\title{
Mobility Management for Vehicular Ad Hoc Networks
}

\author{
Marc Bechler, Lars Wolf \\ Institute of Operating Systems and Computer Networks \\ Technische Universität Braunschweig, Germany \\ Email: [bechler, wolf]@ibr.cs.tu-bs.de
}

\begin{abstract}
Vehicular ad hoc networks are gaining importance for inter-vehicle communication, because they allow for the local communication between vehicles without any infrastructure, configuration effort, and without the high costs of cellular networks. Besides local data exchange, vehicular applications may be extended by accessing Internet services. The access is provided by Internet gateways installed along the roadside. However, the Internet integration requires a respective mobility support of the vehicular ad hoc network. In this paper we propose MMIP6, a communication protocol that integrates multihop IPv6-based vehicular ad hoc networks into the Internet. Whereas existing approaches are focused on small-scale ad hoc networking scenarios, MMIP6 is highly optimized for scalability and efficiency. The evaluation showed that MMIP6 is a suitable solution providing a scalable mobility support with an acceptable performance characteristic.
\end{abstract}

\section{INTRODUCTION}

With the availability of DSRC (dedicated short range communication) technology, multihop ad hoc networks will become a key technology for vehicular environments. In vehicular ad hoc networks (VANETs), vehicles are able to communicate locally without relying on any infrastructure or base stations controlling medium access. An example is the FleetNet communication system, a radio communication technology based on UTRA TDD for ad hoc networking between vehicles [1]. In order to achieve multihop communication, the forwarding of data to the targeted vehicle in VANETs typically uses a location-based ad hoc routing protocol instead of IP addresses [2].

VANETs are very important for the development of vehicular-centered applications such as floating car data where vehicles generate and collect local information, distribute this information locally and consume local information received from neighboring vehicles. These applications are not necessarily limited to inter-vehicle communication. In VANETs, Internet gateways (IGWs) installed at the roadside can provide a temporary Internet access, which opens up the Internet for the VANET and vice versa. This communication scenario is illustrated in fig. 1 where an IGW provides Internet access for the passing vehicles organized in a VANET. This way, vehicular applications can also consider information from the global Internet. An example could be information about the current road and weather conditions along the trip, which can be used for an optimal route guidance.

However, the Internet access requires a respective mobility management to handle the mobility of the vehicles in the

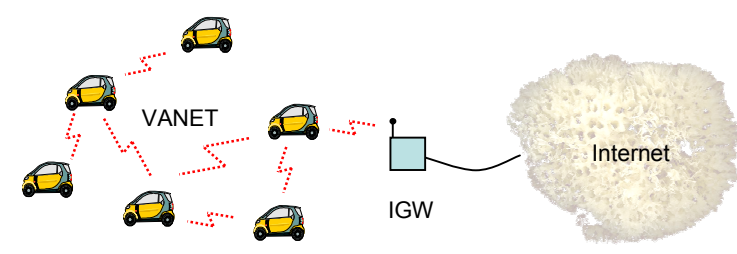

Fig. 1. VANET scenario

Internet. It therefore has to ensure that the requested data from the Internet is always delivered via an appropriate IGW to the vehicle. Vice versa, the vehicles must be able to discover the IGWs within the VANET even when they are multiple hops away. This paper proposes MMIP6, a highly optimized mobility management protocol for VANETs. The following section specifies the requirements for the mobility management of VANETs and gives an overview of related work for the mobility management of multihop ad hoc networks. Section 3 introduces MMIP6, which is evaluated in section 4. Finally, section 5 concludes this paper.

\section{REQUIREMENTS AND RELATED WORK}

Communication in the Internet is based on hierarchical IP addresses with a static (address) topology to route IP packets between communicating peers. In contrast, VANETs are highly mobile: The topology of a VANET changes dynamically and vehicles permanently change their gateway to the Internet. Moreover, vehicles should be accessible from hosts in the Internet independent of their current location. This enables, e.g., the development of applications for remote diagnostics where a vehicle can be inspected through the Internet by a car vendor. The accessibility requires a global IP-based addressing scheme where each vehicle receives a globally routable and permanent IP address. For this task, the 32 bit address space of IPv4 is too small and the deployment of IPv6 is an indispensable requirement since its 128 bit addresses provide sufficient capacity.

The mobility management has to ensure that the requested data from the Internet is always routed via an appropriate IGW to the vehicle. Therefore, the mobility management has to meet the following requirements [3]:

- Seamless Mobility: The VANET should appear as a transparent extension of the Internet. Hence, the mobility of the vehicles must be hided: If an IGW is available, 
communication between vehicle and Internet host must be possible independent of the current position of the vehicle. This also includes handoffs between gateways.

- VANET Characteristics: The mobility management has to support IPv6-based multihop VANETs as well as vehicular characteristics like a potentially high mobility.

- Scalability \& Efficiency: VANETs may become very large comprising potentially thousands of vehicles. Hence, the protocol mechanisms must be highly scalable and efficient in terms of overhead caused by the mobility management.

Mobile IPv6 (RFC 3775) cannot be used for the mobility support of multihop VANETs since it always requires a direct link layer connection between gateway and mobile node [4]. Hence, several approaches were proposed to integrate multihop ad hoc networks into the Internet using Mobile IP. This related work can be classified into three categories: ad hoc routing extensions, multicast extensions, and applicationspecific enhancements.

\section{A. Ad Hoc Routing Extensions}

One possibility for mobility management in ad hoc networks is to extend the ad hoc routing protocol to support Mobile IP protocol mechanisms. Such extensions were currently developed for Mobile IPv4 only. A basic principle of these approaches is to use IP broadcasts to detect foreign agents instead of link-local broadcasts used in Mobile IP. Hence, both agent advertisements and agent solicitations are flooded within the ad hoc network. The routing protocol in the ad hoc network has to be modified accordingly to support the respective IP broadcast functionality as well as to determine whether a host is located inside or outside the ad hoc network. Examples are extensions for DSR and AODV [5]. Other approaches developed a completely new ad hoc routing protocol to support Mobile IP like the Flow-Oriented Routing Protocol FORP [6].

Ad hoc routing protocol extensions are unsuitable for the mobility management of VANETs. They highly depend on the routing protocol deployed in the ad hoc network, and they do not support for Mobile IPv6. Moreover, respective approaches are not scalable, because they require a tunneling within the ad hoc network and a broadcast of agent advertisements and agent solicitations.

\section{B. Multicast Extensions for Mobile IP}

IP multicast enables a location-independent addressing and IP packet delivery to a set of hosts belonging to a multicast group. This way, it can be combined with Mobile IP to support ad hoc networks. The general idea is to use Mobile IP for the mobility support of the mobile nodes, whereas the discovery of gateways within the ad hoc network is based on IP multicast. Examples are MMP (Multicast for Mobility Protocol [7]) and the multicast mobility solution proposed by Tseng et al. [8].

The problem of multicast-based approaches is that they require a multicast support of the ad hoc routing protocol, which is not standardized for location-based ad hoc routing protocols. Moreover, multicast support in ad hoc networks is neither considered as scalable nor as efficient [9].

\section{Application-Specific Enhancements}

Whereas routing protocol extensions and multicast-based approaches are implemented at the network layer, this section discussed application-specific enhancements to deploy Mobile IP in ad hoc networks. For example, Striegel et al. proposed a gateway model for Mobile IPv4, which provides a uniform set of services to mobile nodes [10]. The gateway model is independent of the underlying ad hoc routing protocol, i.e. it works together with existing ad hoc routing protocols as well as with Mobile IPv4: A set of services enable mobile nodes to discover gateways and to register themselves with the foreign agents on the gateways from within the ad hoc network.

Application-specific enhancements are an interesting approach for the mobility management of VANETs. They support the mobility of nodes and they are independent of the ad hoc routing protocol. However, respective approaches do not fit to the requirements for the mobility management of VANETs, because they do not support IPv6. Moreover, using common service discovery protocols for the identification of the gateways is not considered as scalable since their discovery also relies on solicitations and multicast [11].

\section{MMIP6}

In order to handle the mobility of vehicles, we developed a mobility management protocol called MMIP6. MMIP6 is based on the principles of Mobile IPv4, but was designed to support IPv6-based mobile nodes organized in ad hoc networks. In contrast to related approaches, MMIP6 was developed with respect to VANETs, i.e. the protocol mechanisms take into account the requirements for the mobility management of VANETs described in the previous section.

\section{A. Protocol Overview}

Like Mobile IPv6, MMIP6 uses an agent-based system with a home agent (HA) representing a vehicle in the home network (cf. fig. 2). However, MMIP6 is completely different in the basic concept, the addressing, and the protocol mechanisms being deployed. MMIP6 reintroduces foreign agents (FAs) like in Mobile IPv4, which are located at the IGWs. The FA represents the vehicle located in the VANET; this way, it hides the multihop capability of the VANET and the vehicles appear as "common" mobile nodes. A very important feature is that MMIP6 relies on globally routable and permanent IPv6 addresses to identify the vehicles. With the use of FAs, all vehicles participating in the VANET form one logical IPv6 subnet, where the IGWs act as transition points between the VANET and the Internet. The IPv6 addresses can be assigned statically to each vehicle, i.e. they are preconfigured in the communication hardware shipped with the vehicles. In contrast to Mobile IPv6, a vehicle does not receive a valid IPv6 careof address when entering a foreign network. MMIP6 avoids link-local addresses when a vehicle is located in a foreign network. This supersedes the automatic (stateless or stateful) 


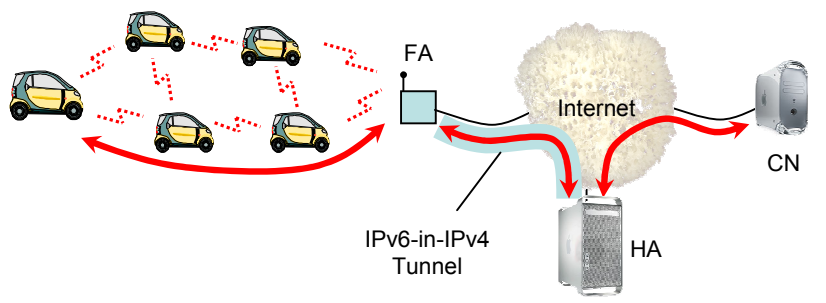

Fig. 2. MMIP6

address configuration of IPv6, which conserves bandwidth in the VANET. Communication in this scenario works as follows:

- If a correspondent node $(\mathrm{CN})$ in the Internet wants to send IP packets to a vehicle $v$, it always transmits them to the $v$ 's home IPv6 address. This way, IP packets are routed via the Internet to the home network of $v$.

- The HA in the home network accepts the IP packets on behalf of $v$ and tunnels them to the FA $v$ is currently registered with. Therefore, MMIP6 uses IPv6-in-IPv4 tunneling as the Internet is still based on IPv4 technology.

- The FA on the IGW unpacks the encapsulated packets and forwards them to $v$ using the VANET routing protocol.

Conversely, a vehicle that wants to send IP packets to a $\mathrm{CN}$ in the Internet first transmits them to the FA. Then, the FA tunnels the IP packets back to the HA, which itself unpacks the IP packets and routes them through the Internet to the $\mathrm{CN}$.

In MMIP6, the VANET routing protocol has to determine the delivery of data. If the receiver is a vehicle $v_{r}$ located in the VANET and can be reached via multihop communication, the VANET routing protocol has to deliver the IP packets locally. Otherwise, the data will be delivered to the FA the sending vehicle $v_{s}$ is currently registered with. This decision process comprises two aspects: As the VANET forms one logical IPv6 subnet, it can be determined in a first step whether or not the receiver is a vehicle within the VANET or whether it is a $\mathrm{CN}$ in the Internet by comparing the subnets addressed. A second aspect optimizes communication in case $v_{r}$ is addressed but cannot be reached with multihop communications. In this situation, the location-based ad hoc routing protocol cannot resolve the position of $v_{r}$ resulting in the following communication path:

1) Vehicle $v_{s}$ delivers the IP packets to its FA, denoted as $\operatorname{FA}\left(v_{s}\right)$, which tunnels the data back to $\operatorname{HA}\left(v_{s}\right)$.

2) As the global IPv6 address of each vehicle belongs to a home network in the Internet, $\mathrm{HA}\left(v_{s}\right)$ forwards the data to $\mathrm{HA}\left(v_{r}\right)$ of the targeted vehicle $v_{r}$.

3) In turn, $\mathrm{HA}\left(v_{r}\right)$ tunnels the data to $\operatorname{FA}\left(v_{r}\right)$, the FA $v_{r}$ is currently registered with.

4) Finally, $\mathrm{FA}\left(v_{r}\right)$ forwards the data to $v_{r}$ over the VANET.

In order to handle the interoperability with the IPv4-based Internet, MMIP6 is integrated into a proxy-based communication architecture. This way, an IPv6-based vehicle is able to access IPv4-based CNs in the Internet.

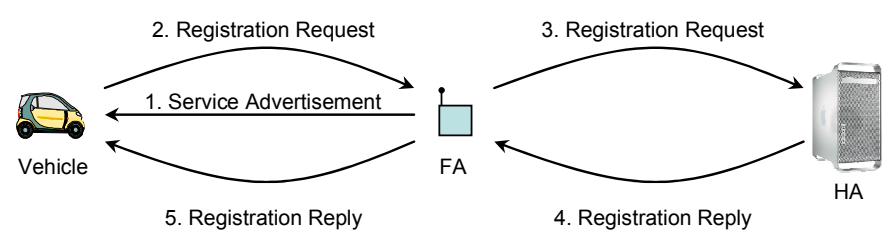

Fig. 3. Registration Procedure in MMIP6

\section{B. Foreign Agent Discovery and Registration}

The FA discovery in MMIP6 is based on a proactive service discovery protocol optimized for large-scale multihop VANETs. The key concept of this service discovery protocol is that FAs do not wait for solicitation messages from vehicles requiring Internet access. Instead, they announce their service periodically. In order to avoid a flooding of the overall ad hoc network, the service announcements are restricted locally to the so-called service area. This can be achieved, e.g., by a hoplimited broadcast or by specifying a geocast region using the geocast capabilities of VANET routing protocols [2]. Vehicles looking for a FA assume a passive role, i.e. they do not discover the FAs actively. Our discovery protocol also supports the selection of the most suitable IGW if several IGWs are available simultaneously. Therefore, we implemented a fuzzybased approach, which considers available information about the gateways [11].

Fig. 3 depicts the situation when a vehicle $v$ moves into the service area of a new IGW. In the first step, $v$ receives the service announcements of the FA and may decide to handoff. In this case, it initiates the registration procedure, where $v$ registers itself through the new FA with its HA. The registration procedure is based on two messages, a registration request and a registration reply: After receiving a service announcement from the IGW in step 1, $v$ sends a registration request to the FA to initiate the registration (step 2). The FA processes this request, updates its internal visitor list, and relays the registration request to the HA of the vehicle (step 3 ). The HA also processes the registration request by updating its mobility bindings and responds with a registration reply message to the FA to grant or to deny the request (step 4). Finally, the FA processes the reply message and relays it to the vehicle (step 5). The registration procedure is similar to the one specified for Mobile IPv4, but requires modified message formats and a different processing in FAs and HAs.

Like in the original Mobile IPv4, registrations have a lifetime in MMIP6, which is specified in the registration request. Before this time expires, the vehicle has to renew its registration with the FA. MMIP6 dynamically determines the lifetime by estimating the time a vehicle travels through the service area of an IGW. In order to avoid vulnerabilities by non-authorized vehicles, registration requests and registration replies can be authenticated. Therefore, each vehicle, FA, and HA support a mobility security association similar to the concept specified for Mobile IPv4. 


\section{EVALUATION}

In contrast to existing approaches, MMIP6 is suitable for large-scale VANETs since it prevents copious transmissions of solicitations/advertisements. The number of solicitations increase with the number of vehicles whereas the proactive discovery of MMIP6 depends on the available IGWs [11]. In existing approaches, the overhead caused by the IGW discovery is correlated with the location of the IGWs since MMIP6 only causes overhead if necessary. In existing approaches, each vehicle permanently has to discover their environment for newly available IGWs even if they are currently not available. Another benefit is that MMIP6 does not require any multicasting capabilities within the ad hoc network. This feature is of specific importance for VANETs, where multicast support is hard to achieve and usually not scalable.

\section{A. Handoff Latency}

The derivation of handoff latency for MMIP6 is based on a mathematical analysis similar to [12]. The handoff latency is the estimated time it takes to handoff to a new IGW in the worst case, where the service areas of two IGWs do not overlap. MMIP6 then has to wait until it first receives and processes the announcements of the new gateway denoted as $t_{A}$. Afterwards, MMIP6 starts the registration procedure, i.e. a registration request is sent to the FA, which takes fwd (RRqst) [hops] multiplied with the transmission delay per link $d_{\text {link }}$. The FA processes the request and relays it to the HA, which takes $t_{\text {relay }}$. The delay for the processing is denoted by the $p$ function. After processing, the HA transmits the registration reply back to the FA, which processes the reply. The FA then has to find the position of the vehicle $\left(t_{\text {loc Query }}\right)$, and finally forwards the reply to the vehicle. Hence, the overall delay for a handoff delay ${ }_{H O}$ is calculated as follows:

$$
\begin{aligned}
\text { delay }_{H O} \approx & t_{A}+f w d(\text { RRqst }) \cdot d_{\text {link }}+p(F A, \text { RRply })+ \\
& t_{\text {relay }}+p(H A)+t_{\text {relay }}+p(F A, R R p l y)+ \\
& t_{\text {locQuery }}+f w d(\text { RRply }) \cdot d_{\text {link }} \\
\leq & \frac{1}{f_{A}}+\frac{l_{\text {geo }} \cdot d_{\text {link }}}{r}+2 \cdot\left(p(F A)+t_{\text {relay }}\right)+ \\
& p(H A)+t_{\text {locQuery }}
\end{aligned}
$$

Thereby, the varying parameter $t_{A}$ is limited by the inverse frequency $f_{A}$ of the announcements. The delay between the targeted vehicle and the IGW is upper-bound by the number of hops between vehicle and IGW defined by the fraction of the service area radius $\left(l_{g e o} / 2\right)$ and radio transmission range $r$. This delay is independent of the routing protocol, because the route in the optimal case is implicitly included in the flooding. The processing delay in the FA is upper-bound by $p(F A)$.

\section{B. Measurements}

In order to evaluate MMIP6, we implemented a prototype for the Linux operating system. The prototype is based on Dynamics Mobile IPv4 implementation, which was modified to provide the MMIP6 functionality. The goal of the evaluation is to investigate the performance of MMIP6 under more

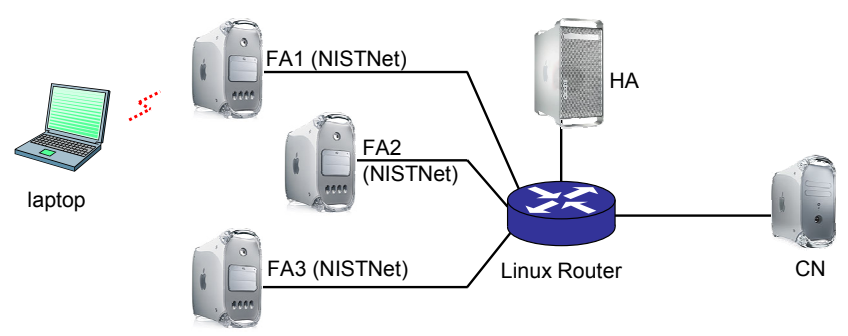

Fig. 4. Testbed used for the evaluation

realistic situations. Scalability is not detailed here since it basically depends on the proactive service discovery, which already proved its scalability [11].

For the measurements, we set up a testbed as illustrated in fig. 4. The testbed contained one $\mathrm{CN}$ connected via a router to three FAs serving different IP subnets and one computer acting as HA. The vehicle was represented by a laptop, which was connected to one of the FAs. In order to study a more realistic ad hoc networking scenario, we used the NISTNet emulation tool to introduce ad hoc networking characteristics. For the evaluation, a data rate of $600 \mathrm{Kbit} / \mathrm{s}$ was used, the delay was $50 \mathrm{~ms}$ with a jitter of $10 \%$ and a packet loss rate of $4 \%$. Although these parameters do not reflect a real world VANET scenario, the emulation is sufficient to investigate the performance behavior of MMIP6 under varying conditions. This configuration also allowed the (theoretical) comparison with standard Mobile IPv6, because in the testbed the laptop is always one physical hop away from the gateway. Although Mobile IPv6 cannot be used to integrate ad hoc networks into the Internet (and does not provide the necessary scalability), it may serve as a theoretical reference for the performance measurements of MMIP6. We measured the throughput of TCP and UDP using MMIP6 and Mobile IP. A handoff was triggered three times: the first two handoffs with a disconnection period of $10 \mathrm{~s}$ and the third (smooth) handoff without a disconnection period. The service advertisement periodicity of MMIP6 was configured to send one service advertisement every $2 \mathrm{~s}$. For the comparison, we configured Mobile IP accordingly, i.e. the MN sends router solicitations every $2 \mathrm{~s}$.

In the first measurement, we tried to find out the number of successfully transmitted IP packets. Therefore, we sent an UDP stream from the laptop to the $\mathrm{CN}$. At the $\mathrm{CN}$, we measured the number of successfully received UDP packets. Fig. 5 shows the results when using Mobile IP and MMIP6. In case of a the first and second handoff with the $10 \mathrm{~s}$ disconnection (the horizontal lines in the graphs), both Mobile IP and MMIP6 perform similarly. In contrast to Mobile IPv6, the third handoff was detected immediately by MMIP6.

Another interesting evaluation is the investigation of the throughput of TCP. Therefore, we used the standard configuration of TCP in Linux. For the measurements, we sent a 1.5 Mbyte file from the $\mathrm{MN}$ to the $\mathrm{CN}$. The measurements illustrated in fig. 6 show an irregular behavior of both MMIP6 and Mobile IP. Whereas Mobile IP has performance benefits 


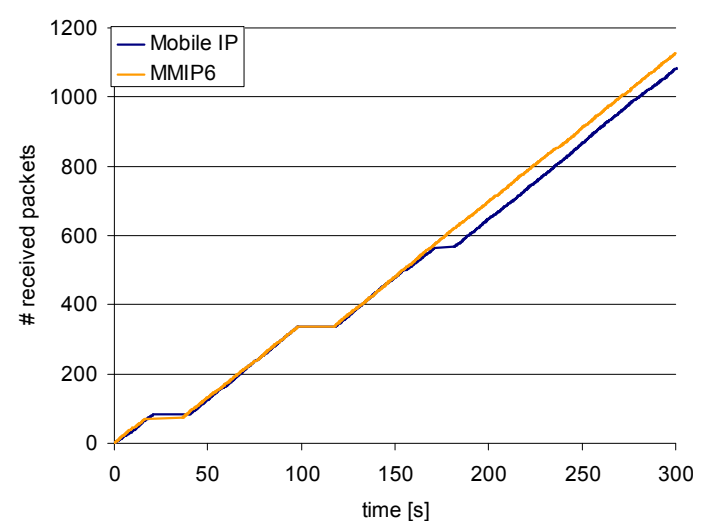

Fig. 5. Evaluation of MMIP6 using UDP

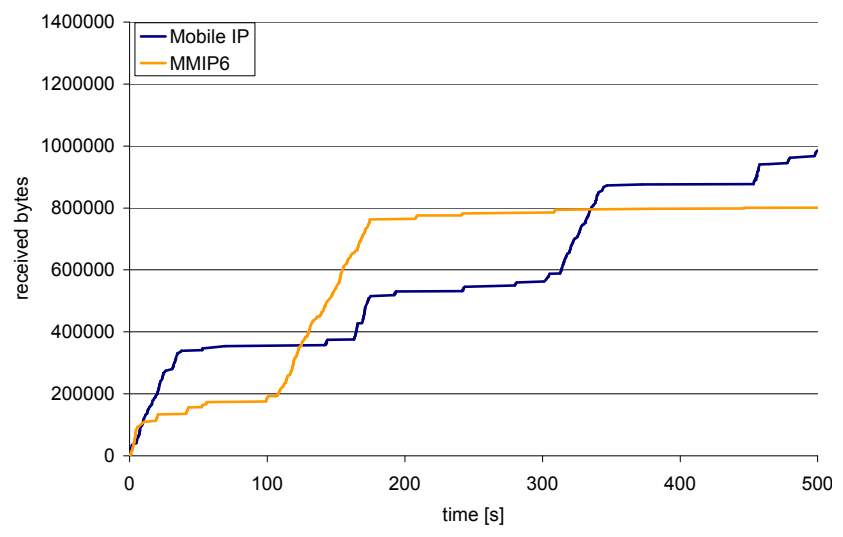

Fig. 6. Evaluation of MMIP6 using TCP

in the beginning (until about 110s) and in the end (from about $330 \mathrm{~s}$ ), MMIP6 outperforms Mobile IP between $110 \mathrm{~s}$ and $330 \mathrm{~s}$. An explanation for this difference is that the measurements highly depend on the unpredictable and statistical behavior of NISTNet. This way, MMIP6 was not able to recover from the second handoff before the third handoff occurred. We therefore carried out our measurements several times and observed that the overall performance of MMIP6 was statistically slightly lower than Mobile IP. Hence, we can carefully conclude that, in contrast to standard Mobile IPv6, MMIP6 is able to integrate ad hoc networks into the Internet without significant performance degradations. However, Mobile IPv6 must be seen as a theoretical reference for the evaluation of MMIP6, because Mobile IPv6 cannot be used to integrate multihop ad hoc networks into the Internet.

\section{CONCLUSION}

Vehicular ad hoc networks will become very important for inter-vehicle communication. Hence, there is a growing need to integrate them seamlessly into the Internet, which is not possible using standard Mobile IP. The MMIP6 protocol proposed in this paper is able to integrate vehicles organized in IPv6-based multihop VANETs into the Internet. In contrast to existing approaches, MMIP6 is optimized for scalability and efficiency. The key concept is a proactive service discovery protocol for Internet gateways providing connectivity to the Internet. This protocol is combined with an optimized mobility management protocol to handle the mobility of the vehicles. Due to the proactive nature of the foreign agent discovery, MMIP6 scales well with the size of the ad hoc network. In order to determine the performance of MMIP6, we made a comparison of MMIP6 with the theoretical performance of Mobile IPv6. The results showed that MMIP6 has similar performance characteristics. As a result, MMIP6 is a suitable approach to integrate large-scale multihop VANETs into the Internet that comes along with an insignificant impact on the communication performance.

Future work comprises an improved evaluation of MMIP6 in different and more realistic ad hoc networking scenarios. However, such an evaluation relies on exact network models, mobility patterns, and network performance metrics which are not yet available. An investigation of the configuration of MMIP6 in such scenarios may further improve the communication efficiency. For example, the periodicity of the announcements determines both overhead and performance. However, this fine tuning also requires realistic models of typical vehicular ad hoc networking scenarios.

\section{REFERENCES}

[1] W. Franz, R. Eberhardt, and T. Luckenbach, "FleetNet - Internet on the Road," in Proceedings of the $8^{\text {th }}$ World Congress on Intelligent Transport Systems, Sydney, Australia, Oct. 2001.

[2] M. Möske, H. Füßler, H. Hartenstein, and W. Franz, "Performance Measurements of a Vehicular Ad Hoc Network," in Proceedings of the $59^{\text {th }}$ IEEE Semiannual Vehicular Technology Conference (VTC), Milan, Italy, May 2004.

[3] C. E. Perkins, Mobile IP Design Principles and Practices. Addison Wesley, 1998.

[4] M. Bechler, W. J. Franz, and L. Wolf, "Mobile Internet Access in FleetNet," in Proceedings of the $13^{\text {th }}$ Fachtagung Kommunikation in Verteilten Systemen (KiVS), Leipzig, Germany, Feb. 2003.

[5] U. Jörnsson, F. Alriksson, T. Larsson, P. Johansson, and G. Q. Maguire Jr., "MIPMANET - Mobile IP for Mobile Ad Hoc Networks," in Proceedings of the $1^{\text {st }}$ ACM International Symposium on Mobile Ad Hoc Networking and Computing (MobiHOC), Boston, Massachusetts, USA, Aug. 2000.

[6] W. Su and M. Gerla, "IPv6 Flow Handoff in Ad Hoc Wireless Networks Using Mobility Prediction," in Proceedings of the 1999 IEEE Global Telecommunications Conference (GLOBECOM), Rio de Janeiro, Brazil, Dec. 1999.

[7] A. Mihailovic, M. Shabeer, and A. H. Aghvami, "Multicast for Mobility Protocol (MMP) for Emerging Internet Networks," in Proceedings of the $11^{\text {th }}$ IEEE International Symposium on Personal, Indoor and Mobile Radio Communications (PIMRC), London, UK, Sept. 2000.

[8] Y.-C. Tseng, C.-C. Shen, and W.-T. Chen, "Integrating Mobile IP with Ad Hoc Networks," IEEE Computer, May 2003.

[9] J. Macker and V. Park, "Anycast Routing for Mobile Networking," in Proceedings of the 1999 IEEE Military Communications Conference (MILCOM), Atlantic City, New Jersey, USA, Nov. 1999.

[10] A. Striegel, R. Ramanujan, and J. Bonney, "A Protocol Independent Internet Gateway for Ad-Hoc Wireless Networks," in Proceedings of the $26^{\text {th }}$ IEEE Conference on Local Computer Networks (LCN), Tampa, Florida, USA, Nov. 2001.

[11] M. Bechler, O. Storz, W. Franz, and L. Wolf, "Efficient Discovery of Internet Gateways in Future Vehicular Communication Systems," in Proceedings of the 57 $7^{\text {th }}$ IEEE Vehicular Technology Conference (VTC), Jeju, Korea, Apr. 2003.

[12] J. Xie and I. E. Akyildiz, "A Distributed Dynamic Regional Location Management Scheme for Mobile IP," in Proceedings of the $21^{\text {st }}$ IEEE Conference on Computer Communications (Infocom), New York, USA, June 2002. 